\title{
Engeletin Protects Against TNF- $\alpha$-Induced Apoptosis and Reactive Oxygen Species Generation in Chondrocytes and Alleviates Osteoarthritis in vivo
}

This article was published in the following Dove Press journal:

Journal of Inflammation Research

\author{
Hao Wang ${ }^{1} *$ \\ Zengxin Jiang ${ }^{1} *$ \\ Zhiying Pang' \\ Guobin $\mathrm{Qi}^{1}$ \\ Bingxuan Hua' \\ Zuoqin Yan' \\ Hengfeng Yuan ${ }^{2}$ \\ 'Department of Orthopaedic Surgery, \\ Zhongshan Hospital, Fudan University, \\ Shanghai, People's Republic of China; \\ ${ }^{2}$ Department of Orthopaedic Surgery, \\ Shanghai Sixth People's Hospital, Shanghai \\ Jiaotong University, Shanghai, People's \\ Republic of China \\ *These authors contributed equally to \\ this work
}

Correspondence: Zuoqin Yan Department of Orthopaedic Surgery, Zhongshan Hospital, Fudan University, 180 Fenglin Road, Xuhui District, Shanghai, 200032, People's Republic of China

Tel +86 I38I8009668

Email yan.zuoqin@zs-hospital.sh.cn

Hengfeng Yuan

Department of Orthopaedic Surgery,

Shanghai Sixth People's Hospital, Shanghai Jiaotong University, 600 Yishan Road,

Xuhui District, Shanghai, 200233, People's

Republic of China

$\mathrm{Tel}+86$ I3917398796

Email yuan.hengfeng@hotmail.com
Purpose: Osteoarthritis (OA) is a progressive disease characterized by pain and impaired joint functions. Engeletin is a natural compound with anti-inflammatory and antioxidant effects on other diseases, but the effect of engeletin on OA has not been evaluated. This study aimed to elucidate the protective effect of engeletin on cartilage and the underlying mechanisms.

Methods: Chondrocytes were isolated from rat knee cartilage, and TNF- $\alpha$ was used to simulate OA in vitro. After treatment with engeletin, the expression of extracellular matrix (ECM) components (collagen II and aggrecan) and matrix catabolic enzymes (MMP9 and MMP3) was determined by Western blotting and qPCR. Chondrocyte apoptosis was evaluated using Annexin V-FITC/PI and flow cytometry. Apoptosis-related proteins (Bax, Bcl-2, and cleaved caspase-3) were evaluated by Western blotting. The mitochondrial membrane potential of chondrocytes was measured with JC-1, and intracellular reactive oxygen species (ROS) levels were determined with DCFH-DA. Changes in signaling pathways (Nrf2, NF- $\kappa \mathrm{B}$ and MAPK) were evaluated by Western blotting. In vivo, anterior cruciate ligament transection (ACLT) was used to induce the rat OA model, and engeletin was administered intraarticularly. The therapeutic effect of engeletin was analyzed by histopathological analysis.

Results: Pretreatment with engeletin alleviated TNF- $\alpha$-induced inhibition of ECM components (collagen II and aggrecan) and upregulation of matrix catabolic enzymes (MMP9 and MMP3). Engeletin ameliorated chondrocyte apoptosis by inhibiting Bax expression and upregulating Bcl-2 expression. Engeletin maintained the mitochondrial membrane potential of chondrocytes and scavenged intracellular ROS by activating the Nrf2 pathway. The NF$\kappa \mathrm{B}$ and MAPK pathways were inhibited by treatment with engeletin. In vivo, ACLT-induced knee OA in rats was alleviated by intraarticular injection of engeletin.

Conclusion: Engeletin ameliorated OA in vitro and in vivo. It may be a potential therapeutic drug for OA.

Keywords: engeletin, osteoarthritis, OA, apoptosis, reactive oxygen species, ROS, mitochondrial membrane potential

\section{Introduction}

Osteoarthritis (OA) has emerged as a public health issue that affected 303 million patients in 2017 , and the prevalence continues to increase each year. ${ }^{1,2}$ The pathogenesis of OA is not fully understood, but various factors, such as being overweight, trauma, senescence and abnormal immune responses, have been shown to 
be related to OA progression. ${ }^{3,4}$ Progressive cartilage destruction, subchondral bone remodeling and synovitis are the characteristics of OA and lead to pain and functional impairments of joints, especially those in the knee, hand and hip. ${ }^{5}$ For these end-stage OA patients, arthroplasty of the involved joints is inevitable. The American Academy of Orthopaedic Surgeons (AAOS) only recommended nonsteroidal anti-inflammatory drugs (NSAIDs) and tramadol for the relief of symptoms. ${ }^{6}$ To date, no pharmacotherapy has been proven to slow or reverse the progression of OA. Therefore, it is urgent to discover effective drugs that preserve cartilage in the treatment of OA.

Reactive oxygen species (ROS) generation and apoptosis are the principal mechanisms involved in the onset and progression of OA. ROS are a series of short-lived oxygen-containing molecules, including hydroxyl radicals $\left(\mathrm{OH}^{-}\right)$, hydrogen peroxide $\left(\mathrm{H}_{2} \mathrm{O}_{2}\right)$, superoxide anion $\left(\mathrm{O}_{2}{ }^{-}\right)$ and nitric oxide (NO). ${ }^{7}$ ROS play central roles in the inflammatory response of chondrocytes. ROS enhance the expression of proinflammatory mediators, including interleukin-1 $\beta$ (IL-1 $\beta$ ), interleukin-6 (IL-6), tumor necrosis factor alpha (TNF- $\alpha$ ) and prostaglandin $\mathrm{E}_{2}\left(\mathrm{PGE}_{2}\right)$. These cytokines inhibit the expression of extracellular matrix (ECM) components such as collagen II and aggrecan, and facilitate the synthesis of ECM catabolic enzymes, including matrix metalloproteinases (MMPs) and a disintegrin and metalloproteinase with thrombospondin motifs (ADAMTS). ${ }^{8-11}$ Excessive ROS accumulation in cells can also alter the mitochondrial membrane potential, initiating apoptosis and leading to cartilage hypocellularity, which destroys cartilage integrity and results in ECM degradation. ${ }^{12}$ These biological processes together contribute to the progression of OA. Therefore, drugs that can scavenge intracellular ROS and alleviate chondrocyte apoptosis have the potential to protect cartilage from degeneration.

Engeletin (dihydrokaempferol 3-rhamnoside) is a naturally occurring compound that is widely distributed in vegetables and fruits. ${ }^{13-16}$ Recent studies have shown that engeletin has anti-inflammatory, anticarcinogenic and antioxidant properties. ${ }^{17-19}$ Engeletin has been proven to be an activator of the nuclear factor erythroid 2-related factor 2 (Nrf2) pathway in BV-2 cells. ${ }^{17} \mathrm{Nrf} 2$ is a key transcription factor that regulates antioxidant processes. Activation of the Nrf2 pathway facilitates the expression of downstream antioxidant proteins, including heme oxygenase-1 (HO-1), superoxide dismutase (SOD) and
$\mathrm{NAD}(\mathrm{P}) \mathrm{H}$ dehydrogenase, quinone 1 (NQO1). ${ }^{20-22}$ The nuclear factor kappa-B (NF- $\kappa \mathrm{B})$ and mitogen-activated protein kinase (MAPK) pathways also participate in modulating chondrocyte growth, differentiation and inflammation. ${ }^{23}$ The progression of $\mathrm{OA}$ is associated with the activation of these two signaling pathways. ${ }^{24}$ Engeletin has been reported to be an inhibitor of the NF$\kappa \mathrm{B}$ and MAPK pathways in many types of cells and tissues. ${ }^{14,15,25,26}$ However, the effect of engeletin on chondrocytes remains unknown.

We hypothesize that engeletin protects chondrocytes from degradation through the Nrf2 pathway activation and NF- $\mathrm{kB}$ and MAPK pathway inhibition. Therefore, in this study, we used TNF- $\alpha$ to simulate chondrocyte inflammation in vitro. ECM component expression, apoptosis, mitochondrial membrane potential, intracellular ROS levels and changes in related signaling pathways were measured after engeletin administration to elucidate the cartilage-protective effects. Moreover, anterior cruciate ligament transection (ACLT) was used to induce knee $\mathrm{OA}$ in rats and engeletin was injected intraarticularly to evaluate its protective effects in vivo.

\section{Materials and Methods Reagents and Antibodies}

Engeletin was purchased from MedChemExpress (Monmouth Junction, NJ, USA) and diluted in dimethyl sulfoxide (DMSO, Sigma-Aldrich, St. Louis, MO, USA). TNF- $\alpha$ was obtained from R\&D Systems (Minneapolis, MN, USA). Cell Counting Kit-8 (CCK-8), protease and phosphatase inhibitor cocktails, RIPA lysis buffer, the nuclear and cytoplasmic protein extraction kit, HRPlabeled goat anti-rabbit $\mathrm{IgG}$, electrochemiluminescence reagents, the Annexin V-FITC apoptosis detection kit and the ROS assay kit were obtained from Beyotime (Shanghai, China). Collagenase type II, DMEM/F-12 culture medium, fetal bovine serum (FBS), $0.25 \%$ trypsin EDTA solution and the penicillin-streptomycin mixture were purchased from Gibco (Grand Island, NY, USA). Reverse transcript master mix and qPCR master mix were obtained from Promega (Madison, WI, USA). Primary antibodies against MMP9, HO-1, NQO1, lamin B1, B-cell lymphoma 2 (Bcl-2), caspase-3 and glyceraldehyde 3-phosphate dehydrogenase (GAPDH) were obtained from Proteintech (Wuhan, China). Antibodies against p38, p-p38, c-Jun N-terminal kinase (JNK), p-JNK, extracellular signal-regulated kinase (ERK), p-ERK, inhibitor kappa 
$\mathrm{B}$ alpha $(\mathrm{I} \kappa \mathrm{B} \alpha), \mathrm{p}-\mathrm{I} \kappa \mathrm{B} \alpha, \mathrm{p} 65, \mathrm{p}-\mathrm{p} 65$ and bcl2-associated $\mathrm{X}$ protein (Bax) were purchased from Cell Signaling Technology (Danvers, MA, USA). Antibodies against collagen II, aggrecan, MMP3, and Nrf2 and the JC-1 mitochondrial membrane potential assay kit were purchased from Abcam (Cambridge, MA, USA).

\section{Experimental Animals}

For primary cell isolation and rat OA model induction, four- and eight-week-old male Sprague-Dawley rats were acquired from the Shanghai SLAC Laboratory Animal Company (Shanghai, China). The protocols for animal experiments were approved by the Ethics Committee of Zhongshan Hospital. The surgeries were performed in accordance with the National Institutes of Health (NIH) Guide for the Care and Use of Laboratory Animals.

\section{Isolation and Culture of Rat Chondrocytes}

Primary chondrocytes were isolated from Sprague-Dawley rats. Four-week-old male Sprague-Dawley rats were euthanized by an overdose injection of sodium pentobarbital. Death was confirmed by checking breath and heartbeat. Verification of death was supplemented by percutaneous cardiac puncture. The articular cartilage was separated from the knee joint and digested with a $0.25 \%$ trypsin EDTA solution for two hours, followed by digestion with $0.1 \%$ collagenase II solution overnight. The isolated chondrocytes were centrifuged, resuspended and cultured in DMEM/F-12 culture medium containing 10\% FBS and a $1 \%$ penicillin-streptomycin mixture in a $37^{\circ} \mathrm{C}$ incubator with $5 \% \mathrm{CO}_{2}$. When the cells reached $80-90 \%$ confluence, chondrocytes were passaged with a trypsin solution. The culture medium was replaced every two days, and chondrocytes between passage one and passage three were used in subsequent experiments.

\section{Cell Viability Assay}

The viability of chondrocytes was determined by CCK-8 assay. Chondrocytes were cultured in 96-well plates at a density of 5000 cells per well and then treated with different concentrations of engeletin $(0 \mu \mathrm{M}, 5 \mu \mathrm{M}, 10$ $\mu \mathrm{M}, 20 \mu \mathrm{M}, 40 \mu \mathrm{M}, 80 \mu \mathrm{M}$, and $160 \mu \mathrm{M}$ ) or DMSO for 24 or 48 hours. After treatment, the culture medium was removed, and the chondrocytes were washed with phosphate-buffered saline (PBS) three times. Then, $100 \mu \mathrm{L}$ of culture medium containing $10 \mu \mathrm{L}$ of CCK-8 solution was added to each well. The plates were incubated at $37^{\circ} \mathrm{C}$ for two hours, and then the absorbance at $450 \mathrm{~nm}$ was determined using a spectrophotometer (FlexStation 3, Molecular Devices, San Jose, CA, USA). To determine the toxicity and IC50 of engeletin, chondrocytes were treated with DMSO or different concentrations of engeletin $(240 \mu \mathrm{M}, 320 \mu \mathrm{M}, 400 \mu \mathrm{M}, 480 \mu \mathrm{M}, 560 \mu \mathrm{M}, 640 \mu \mathrm{M}$, $720 \mu \mathrm{M}, 800 \mu \mathrm{M}, 880 \mu \mathrm{M})$ for 24 hours. CCK-8 was also used to measure the toxicity and the inhibition rate is calculated using the following formula:

Inhibition rate $(\%)=\left(1-A_{\text {test }} / A_{\text {control }}\right) \times 100 \%$

\section{Western Blotting}

Total protein was extracted from chondrocytes using RIPA lysis buffer containing a protease and phosphatase inhibitor cocktail. Nuclear protein and cytoplasmic protein were extracted using a nuclear and cytoplasmic protein extraction kit. Proteins were separated by sodium dodecyl sulfatepolyacrylamide gel electrophoresis (SDS-PAGE) and transferred to polyvinylidene difluoride (PVDF) membranes (Millipore, Bedford, MA, USA). After being blocked with $5 \%$ nonfat milk solution for one hour, the membranes were incubated with the respective primary antibodies overnight and HRP-conjugated secondary antibodies for one hour. Finally, the blots were visualized using enhanced chemiluminescence on an imaging system (Tanon, Shanghai, China).

\section{RNA Isolation and Real-Time PCR}

Total RNA was isolated from chondrocytes using TRIzol reagent (Invitrogen, Carlsbad, CA, USA) according to the manufacturer's protocols. Single-stranded cDNA was synthesized from $1000 \mathrm{ng}$ of the RNA using reverse transcript master mix. Quantitative RT-PCR was performed using a QuantStudio 5 Real-Time PCR System (Applied Biosystems, Foster City, CA, USA) with qPCR master mix. GAPDH was used to normalize the expression using the formula $2-\Delta \Delta \mathrm{Ct}^{27}$ The primer sequences are listed in Table 1.

\section{Detection of Cell Apoptosis}

Chondrocyte apoptosis was examined using an Annexin V-FITC apoptosis detection kit. Chondrocytes were cultured in 6-well plates at a density of 100,000 cells per well. After the respective treatments, the chondrocytes were digested, centrifuged and resuspended in $100 \mu \mathrm{L}$ of binding buffer. The chondrocytes were then incubated with $5 \mu \mathrm{L}$ of Annexin V-FITC and $10 \mu \mathrm{L}$ of propidium iodine (PI) at room temperature for 15 minutes. The chondrocyte 
Table I Primer Sequences Involved in This Research

\begin{tabular}{|l|l|l|}
\hline Name & Forward & Reverse \\
\hline Collagen II & ACGCTCAAGTCGCTGAACAACC & ATCCAGTAGTCTCCGCTCTTCCAC \\
Aggrecan & CTTCCCAACTATCCAGCCAT & TCACACCGATAGATCCCAGA \\
MMP9 & AGGTGCCTCGGATGGTTATCG & TGCTTGCCCAGGAAGACGAA \\
MMP3 & GCTCATCCTACCCATTGCAT & GCTTCCCTGTCATCTTCAGC \\
NQOI & ATCACCAGGTCTGCAGCTTC & GCCATGAAGGAGGCTGCTGT \\
HO-I & ATACCCGCTACCTGGGTGAC & TGTCACCCTGTGCTTGACCT \\
GAPDH & GACAATTTTGGCATCGTGGA & ATGCAGGGATGATGTTCTGG \\
\hline
\end{tabular}

apoptotic rate was measured using a flow cytometer (Accuri C6, BD Biosciences, San Jose, CA, USA).

\section{Detection of Mitochondrial Membrane Potential}

Chondrocyte mitochondrial membrane potential was determined using a JC-1 mitochondrial membrane potential assay. Chondrocytes were seeded in 6-well plates at a density of 100,000 cells per well. After the respective treatments, the chondrocytes were incubated with JC-1 solution for 10 minutes, and images were captured using a confocal laser scanning microscope (FV3000, Olympus, Tokyo, Japan). To quantify changes, chondrocytes were also collected and incubated with JC-1 solution, followed by flow cytometric analysis.

\section{Detection of Intracellular ROS Levels}

The intracellular ROS levels in chondrocytes was measured using a ROS assay kit. Chondrocytes were cultured in 6-well plates at a density of 100,000 cells per well. After the respective treatments, the chondrocytes were digested, collected and resuspended in $10 \mathrm{mM} \mathrm{2} 2^{\prime}, 7^{\prime}-$ dichlorofluorescein diacetate (DCFH-DA) solution. After the cells were incubated in the dark at $37^{\circ} \mathrm{C}$ for 20 minutes, ROS levels were determined using flow cytometry.

\section{Surgical Procedures}

Thirty-six eight-week-old male Sprague-Dawley rats were randomly divided into three equal groups: the control group, OA group and engeletin group. The rat OA model was induced with ACLT. Before the surgery, rats were anesthetized by sodium pentobarbital injection. After anesthesia induction, a longitudinal medial parapatellar incision was made on the unilateral knee followed by transection of the anterior cruciate ligament. Rats in OA and engeletin groups received ACLT surgery while rats in the control group received the same incision without further surgery. After four weeks, rats in engeletin group received intraarticular injections of engeletin once a week (dissolved in normal saline, $100 \mu \mathrm{L}$ containing $50 \mu \mathrm{g}$ engeletin per injection). Rats in the control and OA groups received the same volume of normal saline.

\section{Histopathological Analysis}

After four or eight weeks of injection, six rats in each group were euthanized by an overdose injection of sodium pentobarbital. Knee joints were collected and fixed in 4\% paraformaldehyde for 24 hours, followed by decalcification in $10 \%$ EDTA solution for one month. After decalcification, the tissues were then dehydrated, embedded in paraffin and coronally cut into $3-\mu \mathrm{m}$ slices. The slices were then stained with hematoxylin-eosin (HE), safranin $\mathrm{O} /$ fast green and toluidine blue (TB). The severity of OA was evaluated using the Osteoarthritis Research Society International (OARSI) scoring system. ${ }^{28}$ We used the semi-quantitative grading method reported in this study. The score is the multiplication of grade $(0-6)$ and stage $(0-4)$ and the range is between 0 and 24. Scoring was performed in a blinded manner.

\section{Statistical Analysis}

All experiments were performed at least three independent times. All data are represented as the mean \pm standard deviation (SD). Analysis of the flow cytometry data was performed using FlowJo 10 software (FlowJo, LLC, Ashland, OR, USA). Statistical analyses were performed using GraphPad Prism 8 (GraphPad Software, Inc., La Jolla, CA, USA). Difference between multiple groups were analyzed using one-way analysis of variance (ANOVA) followed by Tukey's post hoc test. P-values < 0.05 were considered statistically significant. 


\section{Results}

\section{Effects of Engeletin on Chondrocyte Viability}

Figure 1A shows the molecular structure of engeletin. To determine the cytotoxicity of engeletin, we used a CCK-8 assay to evaluate cell viability. As shown in Figure $1 \mathrm{~B}$ and $\mathrm{C}$, chondrocytes were treated with various concentrations of engeletin for 24 or 48 hours. Treatment with engeletin at concentrations between $5 \mu \mathrm{M}$ and $160 \mu \mathrm{M}$ improved chondrocyte viability and no significant differences were found among these groups. To determine the IC50 of engeletin, we used higher concentrations of engeletin to treat chondrocytes for 24 hours and inhibition rate was calculated. As shown in Figure 1D, the inhibition rate curve was created using nonlinear regression ("Sigmoidal, 4PL, X is concentration" method provided by GraphPad Prism) and $336 \mu \mathrm{M}$ is the IC50 of engeletin.

\section{Engeletin Alleviated TNF- $\alpha$-Induced Matrix Degradation}

Chondrocytes were cultured in 6-well plates at a density of 100,000 cells per well and pretreated with different concentrations of engeletin $(10 \mu \mathrm{M}$ and $20 \mu \mathrm{M})$ for two hours. Then TNF- $\alpha(20 \mathrm{ng} / \mathrm{mL})$ was added to incubate for another 24 hours. As shown in Figure 2A-I, TNF- $\alpha$ treatment promoted MMP9 and MMP3 expression and suppressed collagen II and aggrecan expression at the mRNA and protein levels. Engeletin pretreatment reversed the effect of TNF- $\alpha$ treatment.

\section{Engeletin Inhibited TNF- $\alpha$-Induced Chondrocyte Apoptosis}

Chondrocytes were pretreated with different concentrations of engeletin (10 $\mu \mathrm{M}$ and $20 \mu \mathrm{M})$ for two hours. TNF- $\alpha(20 \mathrm{ng} / \mathrm{mL})$ was then added to incubate for another 24 hours. Apoptosis was examined using Annexin V-FITC/PI and flow cytometry. As shown in Figure 3A and $\mathrm{B}, \mathrm{TNF}-\alpha$ treatment upregulated the chondrocyte apoptosis, while engeletin pretreatment dose-dependently reversed this effect.

To determine the underlying molecular mechanism by which engeletin inhibits apoptosis, we used Western blotting to measure the expression of apoptosis-related proteins. As shown in Figure $3 \mathrm{C}-\mathrm{F}, \mathrm{TNF}-\alpha$ treatment
A

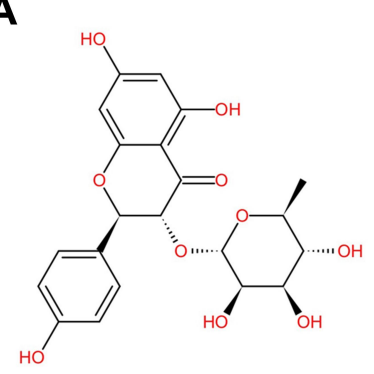

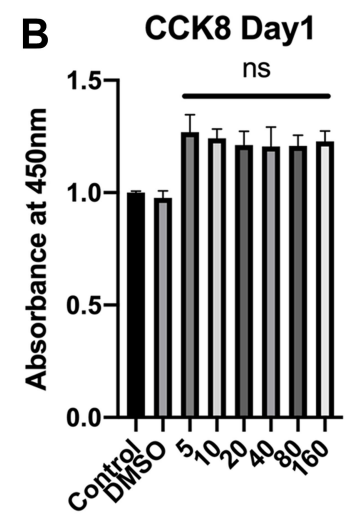

D

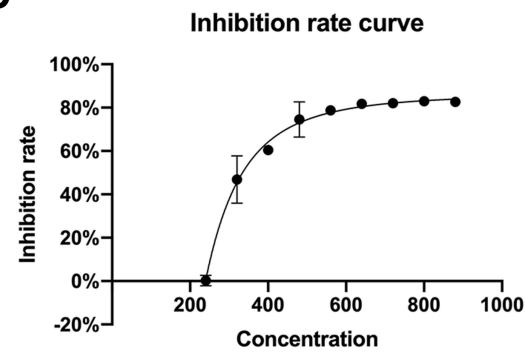

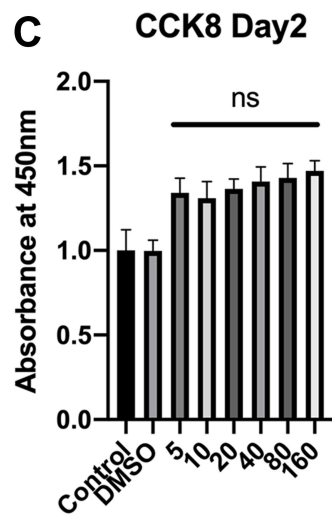

Figure I The molecular structure of engeletin and the effects of engeletin on chondrocyte viability. (A) The molecular structure of engeletin. (B, C) Chondrocytes were treated with DMSO or different concentrations of engeletin $(5,10,20,40,80$ and I60 $\mu$ M) for 24 (B) or 48 (C) hours. Then, cell viability was measured by CCK-8 assay. (D) Chondrocytes were treated with DMSO or different concentrations of engeletin $(240,320,400,480,560,640,720,800,880 \mu \mathrm{M})$ for 24 hours to determine the toxicity of engeletin. The data are presented as the mean \pm SD. $n=3$. ns: not significant. 
A

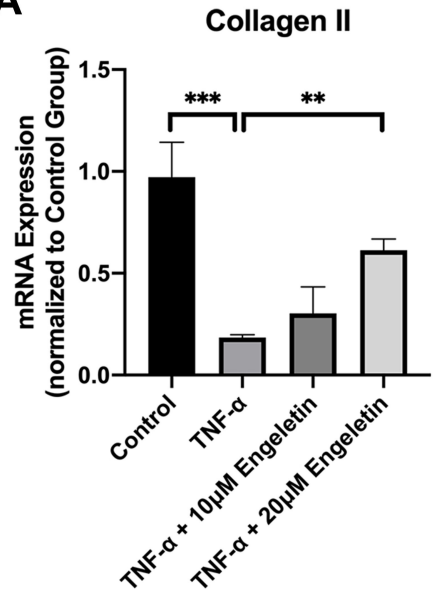

D

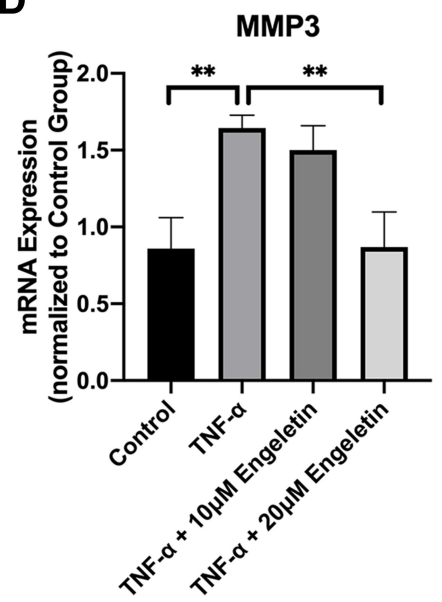

G

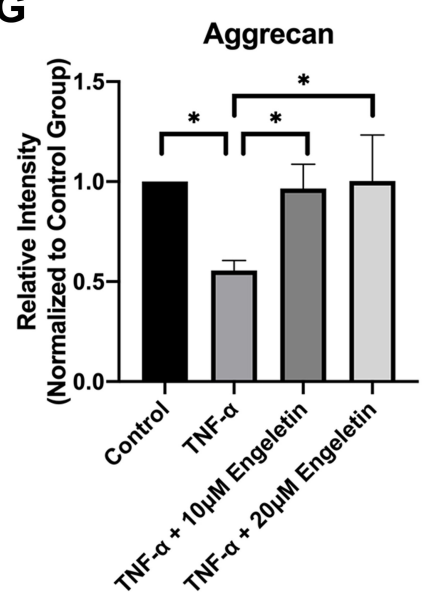

B

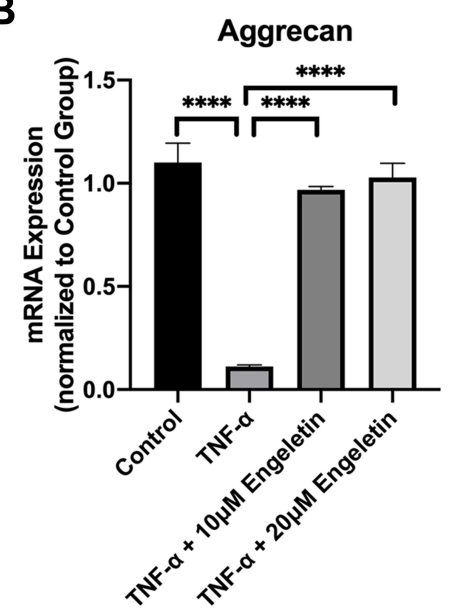

E

Collagen II

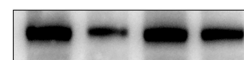

$141 \mathrm{kD}$

Aggrecan

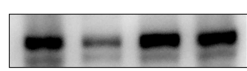

$110 \mathrm{kD}$
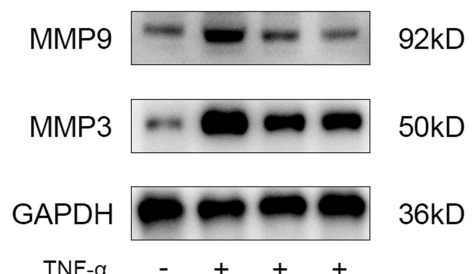

$36 \mathrm{kD}$

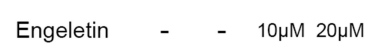

H

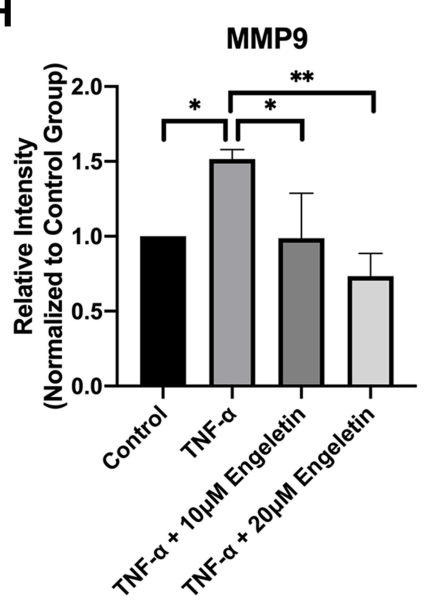

C

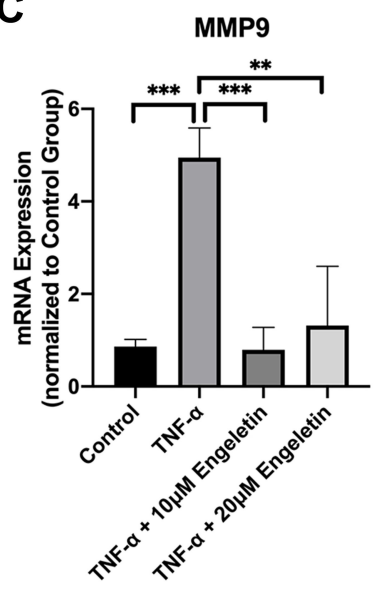

$\mathbf{F}$
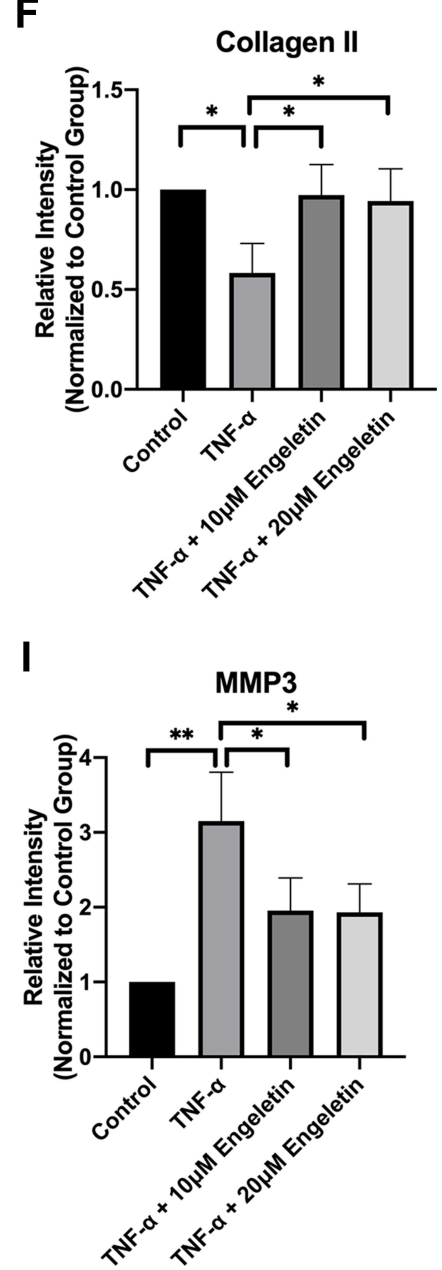

Figure 2 Engeletin alleviated TNF- $\alpha$-induced ECM degradation. Chondrocytes were pretreated with engeletin (10 $\mu$ M and $20 \mu \mathrm{M})$ for two hours, and then TNF- $\alpha$ ( 20 ng/ $\mathrm{mL}$ ) was added to the culture medium and incubated for another 24 hours. (A-D) The relative mRNA expression levels of collagen II, aggrecan, MMP9 and MMP3 were determined by qPCR. (E-I) The protein expression levels of collagen II, aggrecan, MMP9 and MMP3 were determined by Western blotting. The results were quantified using ImageJ software. Protein expression was normalized to GAPDH. The data are presented as the mean \pm SD. $n=3 .{ }^{*} p<0.05, * * p<0.01, * * * p<0.001, * * * * p<0.000 I$.

upregulated the expression of Bax and cleaved caspase-3 and suppressed the expression of Bcl-2, while engeletin pretreatment dose-dependently reversed the effect of TNF- $\alpha$.

\section{Engeletin Maintained Chondrocyte}

\section{Mitochondrial Membrane Potential}

Chondrocytes were pretreated with different concentrations of engeletin $(10 \mu \mathrm{M}$ and $20 \mu \mathrm{M})$ for two hours. 
A
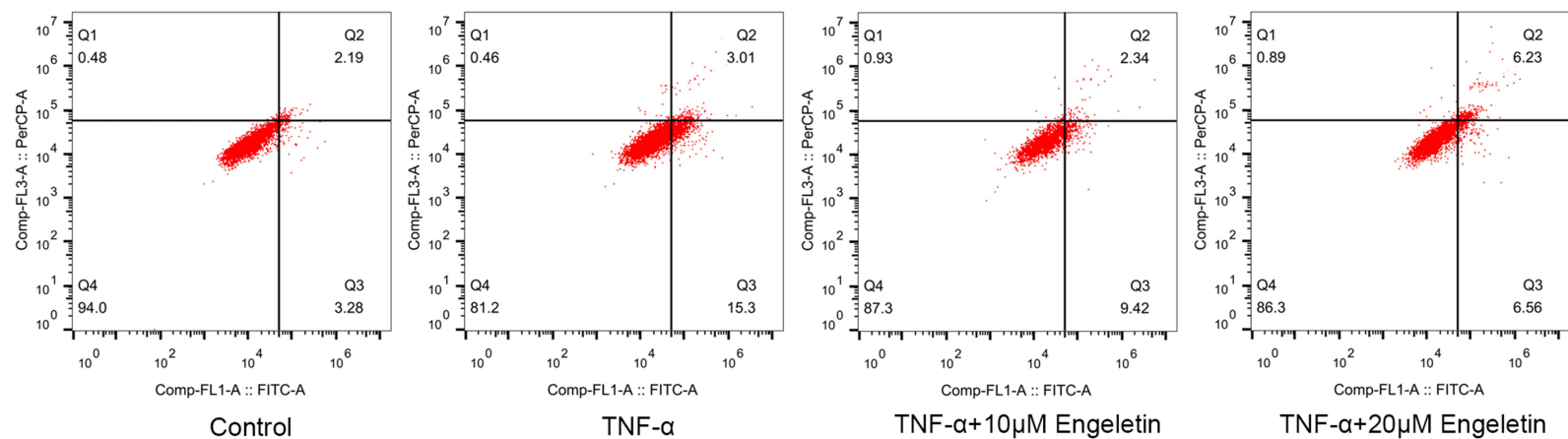

TNF- $\alpha+10 \mu \mathrm{M}$ Engeletin

TNF- $\alpha+20 \mu \mathrm{M}$ Engeletin

B

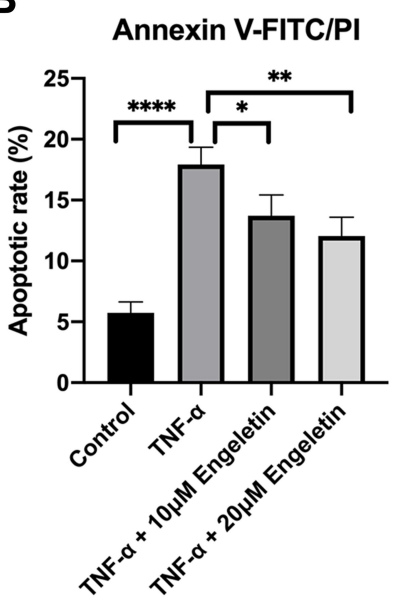

E

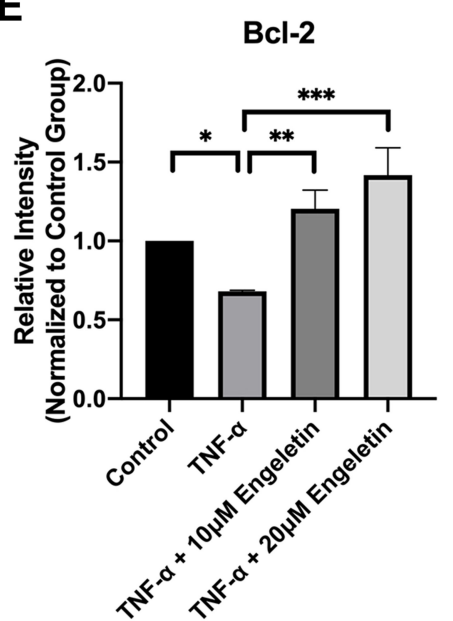

C

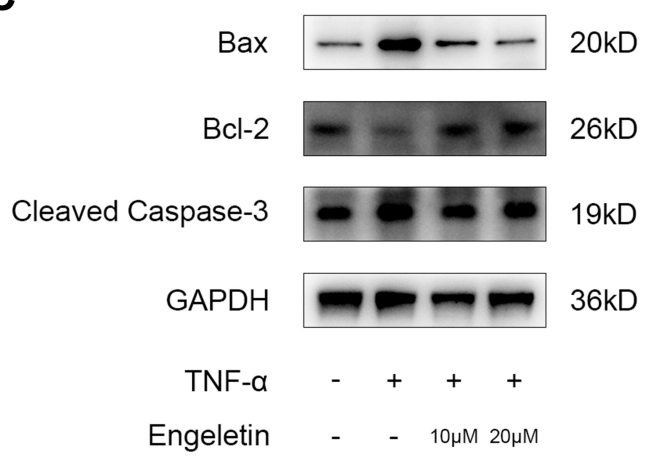

D

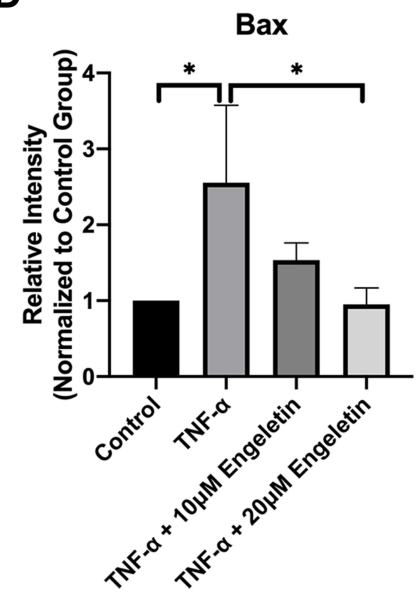

F

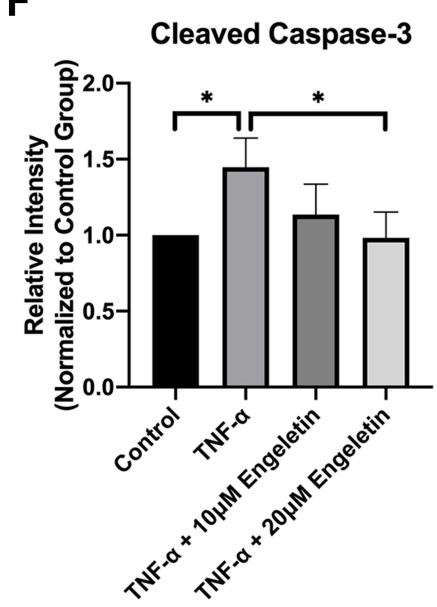

Figure 3 Engeletin ameliorated TNF- $\alpha$-induced chondrocyte apoptosis. Chondrocytes were pretreated with engeletin (I0 $\mu$ M and $20 \mu \mathrm{M})$ for two hours, and then TNF- $\alpha$ $(20 \mathrm{ng} / \mathrm{mL})$ was added to the culture medium and incubated for another 24 hours. (A) Chondrocytes were stained with Annexin V-FITC/PI and measured by flow cytometry to determine the apoptotic rate. (B) Statistical analysis of the apoptotic rate of chondrocytes. (C-F) The protein expression levels of Bax, Bcl-2, and cleaved caspase-3 were determined by Western blotting. The results were quantified using Image software. Protein expression was normalized to GAPDH. The data are presented as the mean \pm SD. $n=3 . *_{p}<0.05, * *_{p}<0.01, * * *_{p}<0.001, * * * * p<0.0001$.

TNF- $\alpha(20 \mathrm{ng} / \mathrm{mL})$ was then added to incubate for another 24 hours. Chondrocytes were then incubated with JC-1 solution and observed using a confocal laser scanning microscope. Figure 4A shows that the red fluorescence was weakened and the green fluorescence was intensified by TNF- $\alpha$ treatment, while engeletin pretreatment dosedependently reversed these effects.

Flow cytometry was also used to quantify the mitochondrial membrane potential. As shown in Figure $4 \mathrm{~B}$ and $\mathrm{C}$, abnormal chondrocytes with collapsed mitochondrial 
A
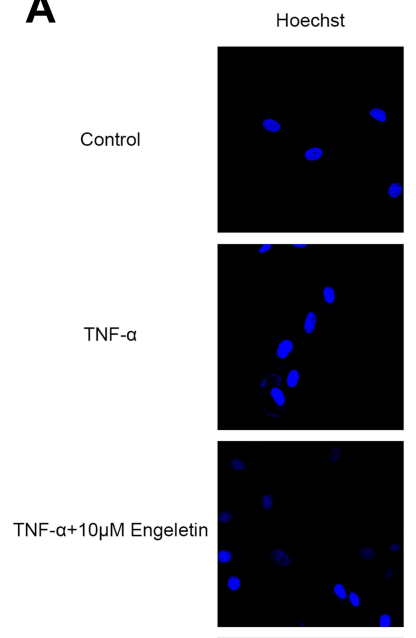

TNF- $\alpha+20 \mu M$ Engeletin
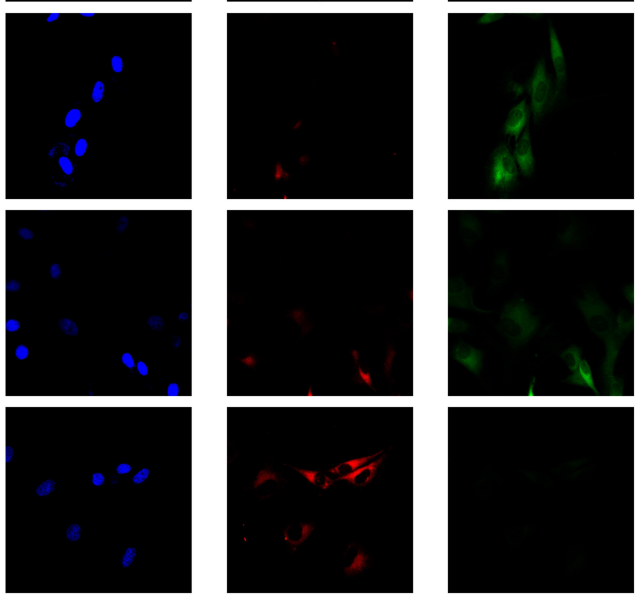

B

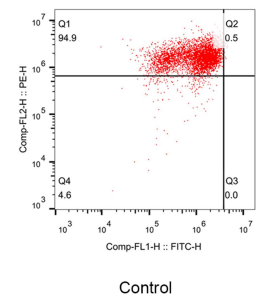

C

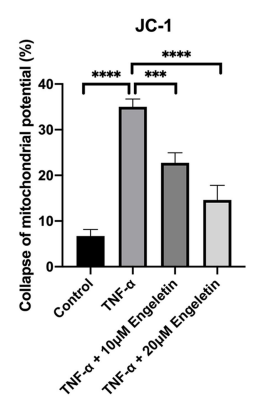

E

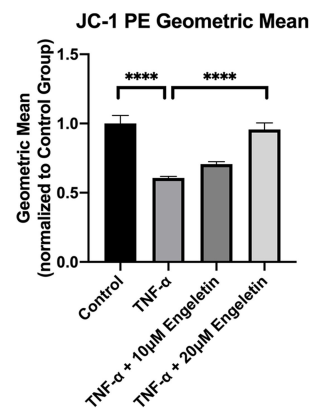

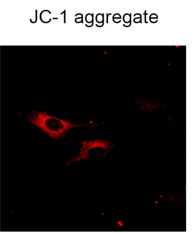
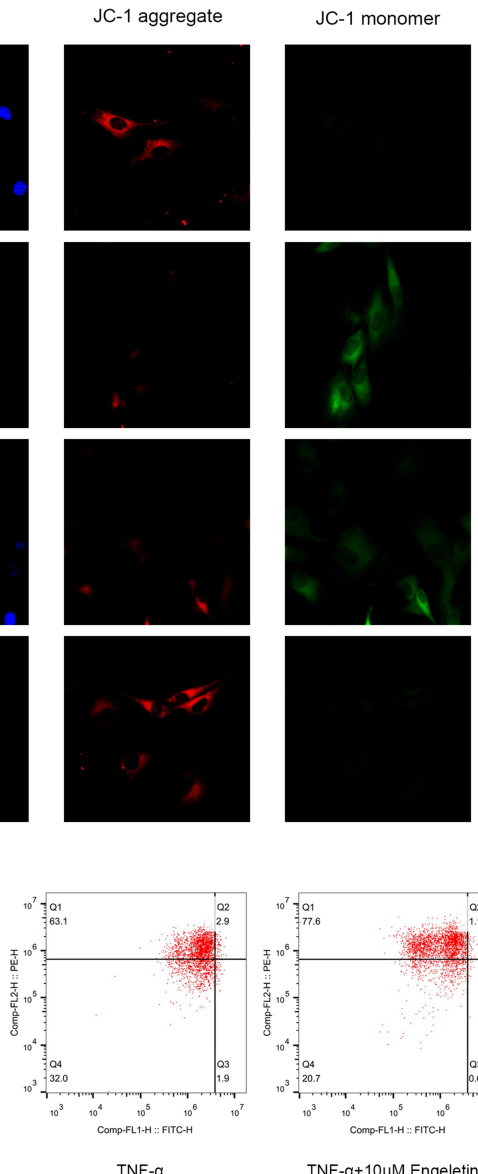

TNF- $\alpha$

D

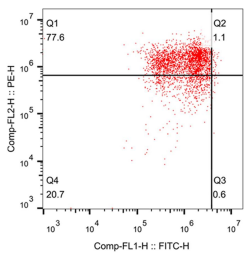

TNF- $\alpha+10 \mu M$ Engeletin
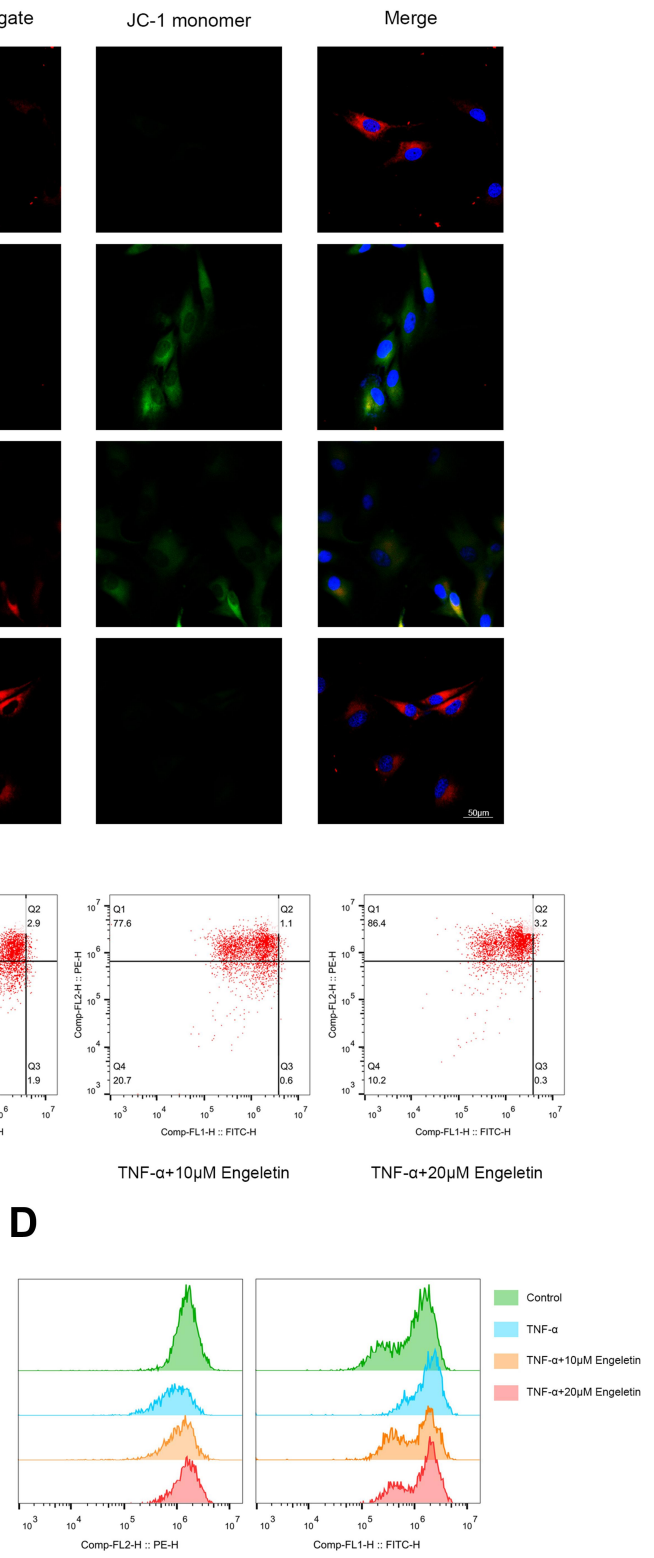

F JC-1 FITC Geometric Mean

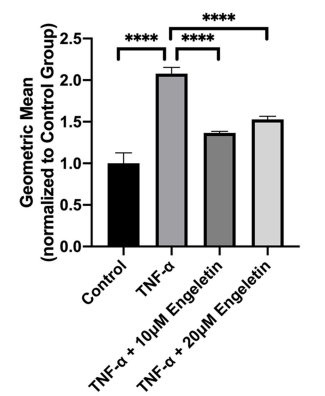

Figure 4 Engeletin maintained the mitochondrial membrane potential of chondrocytes. Chondrocytes were pretreated with engeletin ( $10 \mu \mathrm{M}$ and $20 \mu \mathrm{M})$ for two hours, and then TNF- $\alpha(20 \mathrm{ng} / \mathrm{mL})$ was added to the culture medium and incubated for another 24 hours. (A) Chondrocytes were stained with JC-I and observed using a confocal laser scanning fluoroscope. (B) Chondrocytes were stained with JC-I and measured by flow cytometry. (C) The proportion of the chondrocytes with collapsed mitochondrial potential (Q2+Q3+Q4). (D-F) Quantification of red and green fluorescence. The data are presented as the mean \pm SD. $\mathrm{n}=3$. $* * * \mathrm{p}<0.00 \mathrm{I}$, $* * * * \mathrm{p}<0.000 \mathrm{I}$. 
potential were increased in the TNF- $\alpha$ treatment group, while engeletin pretreatment dose-dependently reversed this effect. The red and green fluorescence intensities were also quantified, and the results are shown in Figure 4D-F. TNF- $\alpha$ treatment intensified the green fluorescence and weakened the red fluorescence, while engeletin dosedependently alleviated these effects. These results indicated that engeletin could maintain the mitochondrial membrane potential.

\section{Engeletin Ameliorated TNF- $\alpha$-Induced ROS Generation in Chondrocytes}

Chondrocytes were pretreated with different concentrations of engeletin $(10 \mu \mathrm{M}$ and $20 \mu \mathrm{M})$ for two hours. TNF- $\alpha(20 \mathrm{ng} / \mathrm{mL})$ was then added to incubate for another 24 hours. Intracellular ROS levels were evaluated using DCFH-DA. Figure 5A and B shows that TNF- $\alpha$ treatment increased intracellular ROS generation, while engeletin pretreatment dose-dependently reversed this effect.

\section{Engeletin Activated Nrf2 Pathway}

The Nrf2 pathway is closely related to intracellular ROS generation and elimination. Chondrocytes were pretreated with different concentrations of engeletin $(10 \mu \mathrm{M}$ and 20 $\mu \mathrm{M})$ for two hours. TNF- $\alpha(20 \mathrm{ng} / \mathrm{mL})$ was then added to incubate for another 24 hours. Figure $5 \mathrm{C}-\mathrm{E}$ shows that engeletin treatment enhanced the nuclear translocation of Nrf2. As shown in Figure 5F-J, engeletin treatment upregulated the expression of NQO1 and HO-1 at the mRNA and protein levels in a dose-dependent manner.

\section{Engeletin Inhibited TNF- $\alpha$-Induced Activation of the NF- $\kappa B$ and MAPK Pathways}

As previously described, recent studies have shown that $\mathrm{NF}-\kappa \mathrm{B}$ and MAPK pathway activation is closely associated with the progression of OA. ${ }^{23,24}$ To determine the effect of engeletin on these pathways, chondrocytes were pretreated with different concentrations of engeletin (10 $\mu \mathrm{M}$ and $20 \mu \mathrm{M})$ for two hours. TNF- $\alpha(20 \mathrm{ng} / \mathrm{mL})$ was then added to incubate for another six hours.

Figure $6 \mathrm{~A}-\mathrm{C}$ shows that engeletin alleviated $\mathrm{TNF}-\alpha-$ induced phosphorylation of $\mathrm{I} \kappa \mathrm{B} \alpha$ and $\mathrm{p} 65$, indicating that engeletin reversed TNF- $\alpha$-induced activation of the NF- $\kappa B$ pathway. Figure $6 \mathrm{D}-\mathrm{G}$ shows that engeletin ameliorated TNF- $\alpha$-induced phosphorylation of ERK, JNK and p38, indicating that engeletin also inhibited TNF- $\alpha$-induced activation of the MAPK pathway.

\section{Engeletin Alleviated Rat OA in vivo}

To evaluate the effect of engeletin on OA in vivo, we used ACLT to induce OA in rats, and engeletin was intraarticularly administered once a week. After four or eight weeks of treatment, the rats were euthanized, and knee joints were collected for histopathological analysis.

Figure $7 \mathrm{~A}$ is the result of engeletin intervention for four weeks. $\mathrm{HE}$, safranin $\mathrm{O} /$ fast green and $\mathrm{TB}$ staining indicated that ACLT treatment resulted in superficial cartilage erosion and the loss of proteoglycan. Four weeks of engeletin administration partially alleviated the destructive effect of ACLT. Figure 7B represents the result of engeletin injection for eight weeks. The protective effect of engeletin was also obvious, but the cartilage destructions in OA group and engeletin group were worse than those of four weeks. The severity of $\mathrm{OA}$ was quantified using the OARSI scoring system, and the results are shown in Figure 7C. The scores of the engeletin group were lower than those of the OA group at both time points. The scores of the OA and engeletin groups at eight weeks were higher than those at four weeks. These results indicated that engeletin could slow down the ACLT-induced progression of OA.

\section{Discussion}

OA has become a worldwide medical burden, and thus far, there have been no available preventive or therapeutic biological interventions. ${ }^{29}$ This study aimed to discover a therapeutic drug for OA. Chondrocytes were treated with TNF- $\alpha$ to mimic the inflammatory environment, and engeletin was then administered. The in vitro findings indicated that engeletin alleviated TNF- $\alpha$ induced ECM degradation, apoptosis, mitochondrial membrane potential destruction and ROS generation in chondrocytes. The cartilage-protective effect occurred through Nrf2 pathway activation and NF- $\mathrm{B}$ and MAPK pathway inhibition. Engeletin was also injected intraarticularly into a rat knee OA model, and histopathological analysis proved that this treatment ameliorated OA in vivo. Figure 8 shows the schematic diagram of the mechanisms. This schematic diagram is created with BioRender.com.

Excessive chondrocyte apoptosis has been proven to be the key factor in OA. Studies have shown that the apoptotic rate of $\mathrm{OA}$ chondrocytes can reach a maximum of $28 \%$, 
A
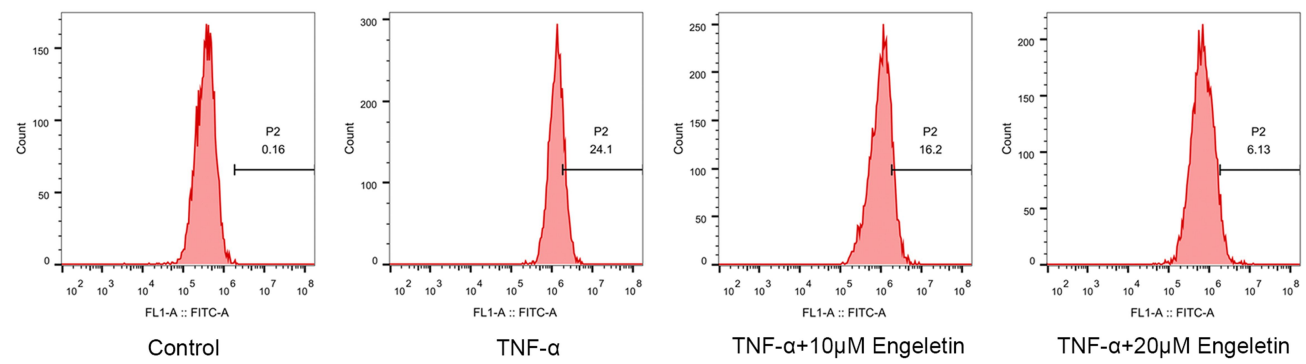

TNF- $\alpha+10 \mu M$ Engeletin

B

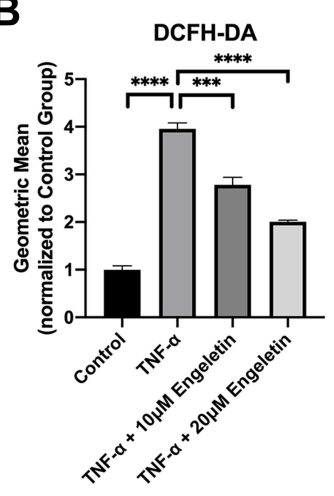

E

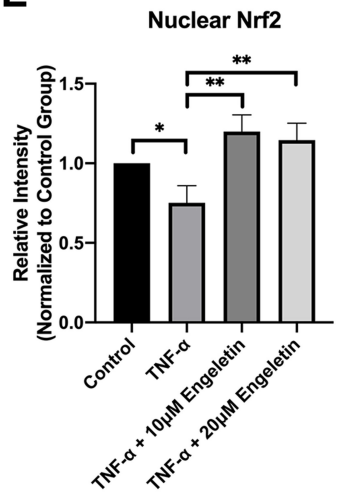

H

NQO1 $-31 \mathrm{kD}$

HO-1 $23 \mathrm{kD}$

GAPDH $36 \mathrm{kD}$

TNF- $\alpha-+++$

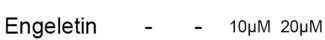

C

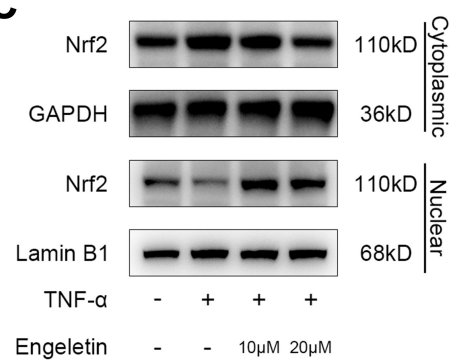

F

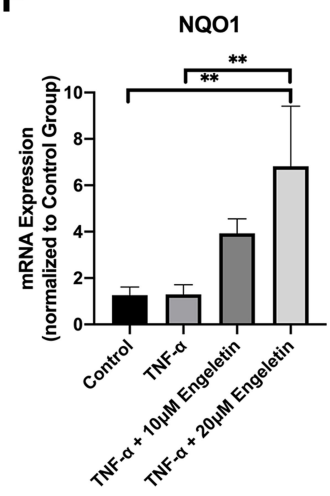

I

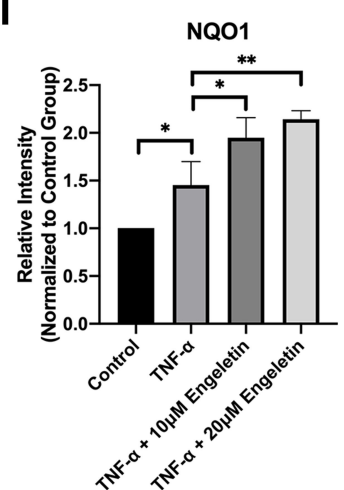

D

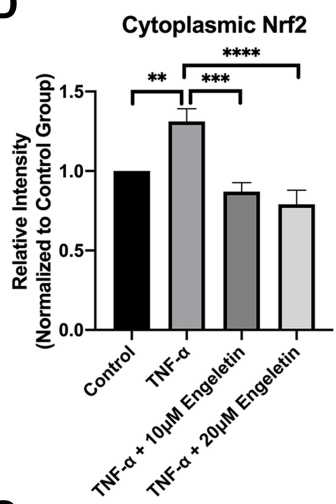

G

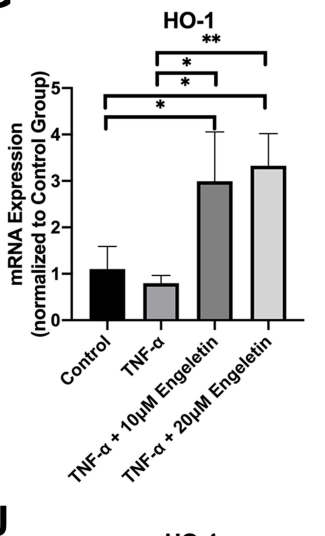

$J$

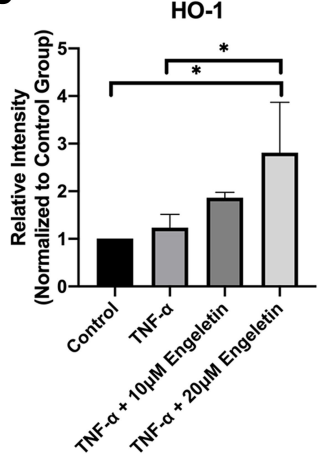

Figure 5 Engeletin alleviated TNF- $\alpha$-induced ROS generation by activating the Nrf2 pathway. Chondrocytes were pretreated with engeletin (I0 $\mu \mathrm{M}$ and $20 \mu \mathrm{M})$ for two hours, and then TNF- $\alpha(20 \mathrm{ng} / \mathrm{mL})$ was added to the culture medium and incubated for another 24 hours. (A) Chondrocytes were stained with DCFH-DA and measured by flow cytometry. (B) The geometric mean of the fluorescence. (C-E) The protein expression levels of nuclear and cytoplasmic Nrf2 were determined using Western blotting. The results were quantified with Imagej. Nuclear protein expression was normalized to Lamin BI and cytoplasmic protein expression was normalized to GAPDH. (F, G) The relative mRNA expression levels of $\mathrm{NQOI}$ and $\mathrm{HO}-\mathrm{I}$ were measured by qPCR. $(\mathrm{H}-\mathrm{J})$ The protein expression levels $\mathrm{NQO}$ and $\mathrm{HO}-\mathrm{I}$ were measured by Western blotting. The results were quantified with ImageJ. Protein expression was normalized to GAPDH. The data are presented as the mean \pm SD. $n=3$. *p $<0.05$, $* * p<0.0$ I, $* * * p<0.00$ I, $* * * * p<0.0001$. 
A

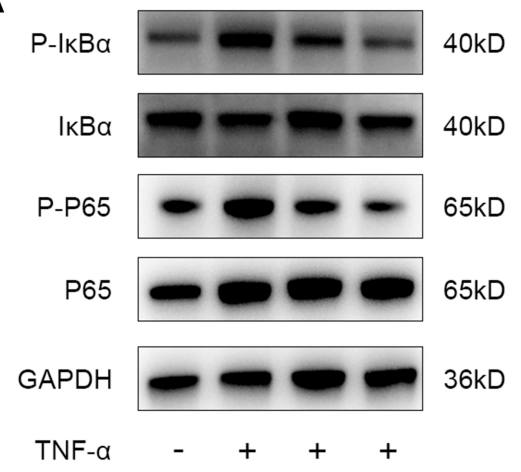

Engeletin - $\quad-10 \mu \mathrm{M} 20 \mu \mathrm{M}$

D

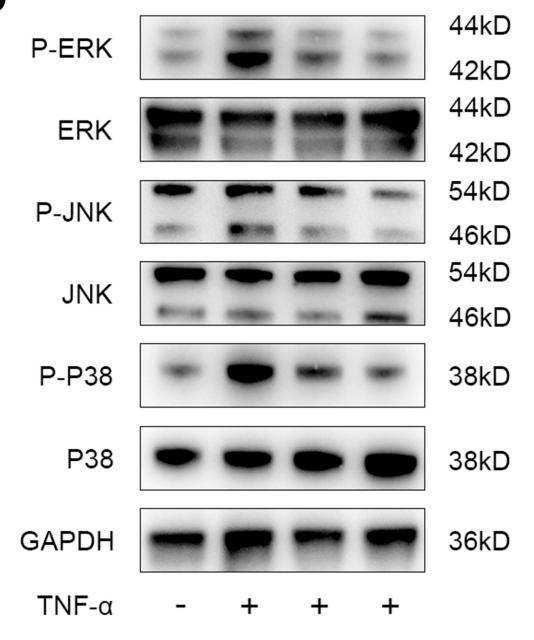

Engeletin - $\quad-10 \mu \mathrm{M} 20 \mu \mathrm{M}$
B

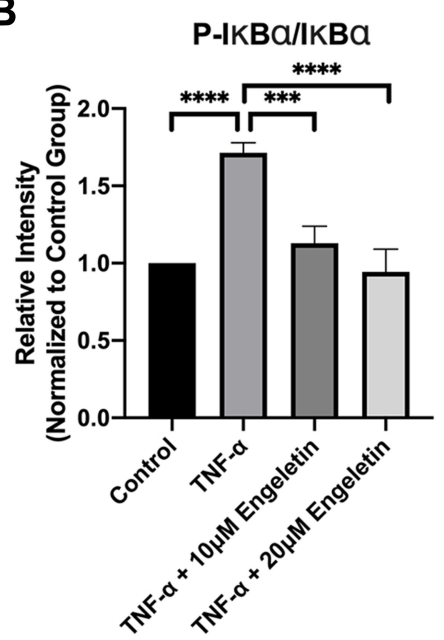

E

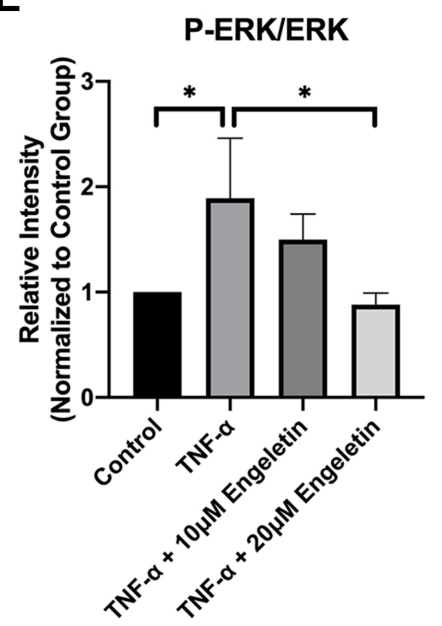

G

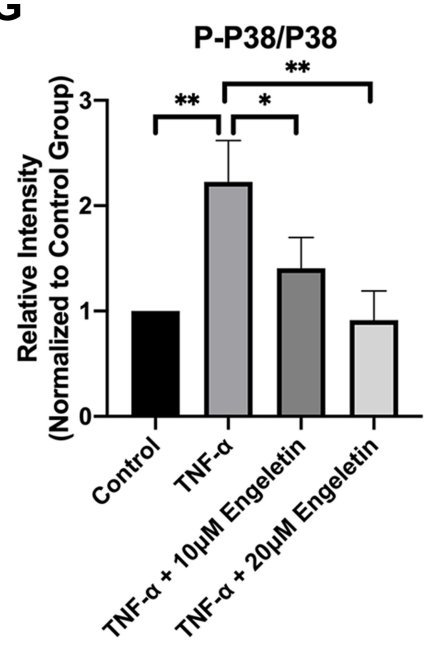

C

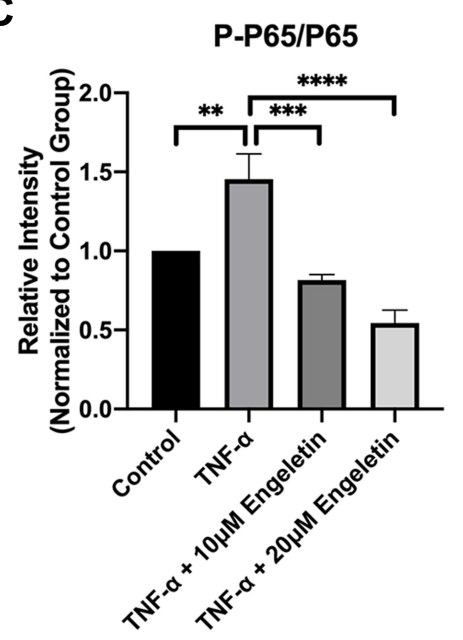

F

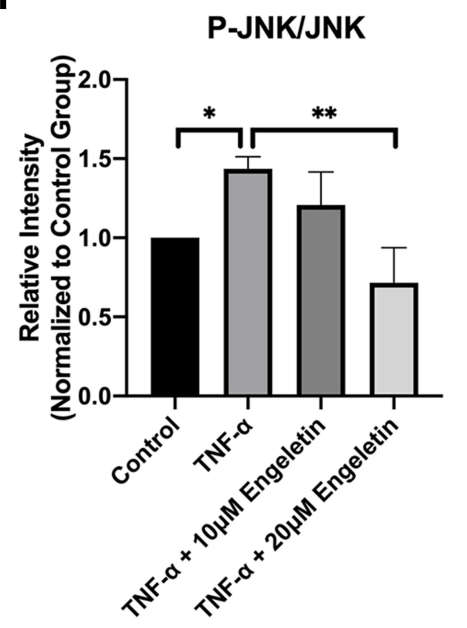

Figure 6 Engeletin inhibited TNF- $\alpha$-induced activation of the NF- $k B$ and MAPK pathways. Chondrocytes were pretreated with engeletin (I0 $\mu$ M and $20 \mu M)$ for two hours, and then TNF- $\alpha(20 \mathrm{ng} / \mathrm{mL})$ was added to the culture medium and incubated for another six hours. (A-C) The protein expression levels of $p-1 \kappa B \alpha$, I $k B \alpha$, $p$-p65 and p65 (components of the NF-kB pathway) were determined by Western blotting. The results were quantified with Image. (D-G) The protein expression levels of $\mathrm{p}$-ERK, ERK, p-JNK, JNK, p-p38 and p38 (components of the MAPK pathway) were evaluated by Western blotting. The results were quantified with Image. The data are presented as the mean \pm SD. $n=3 . *_{p}<0.05, * * p<0.01, * * * p<0.001$, ****p $<0.0001$. 
A

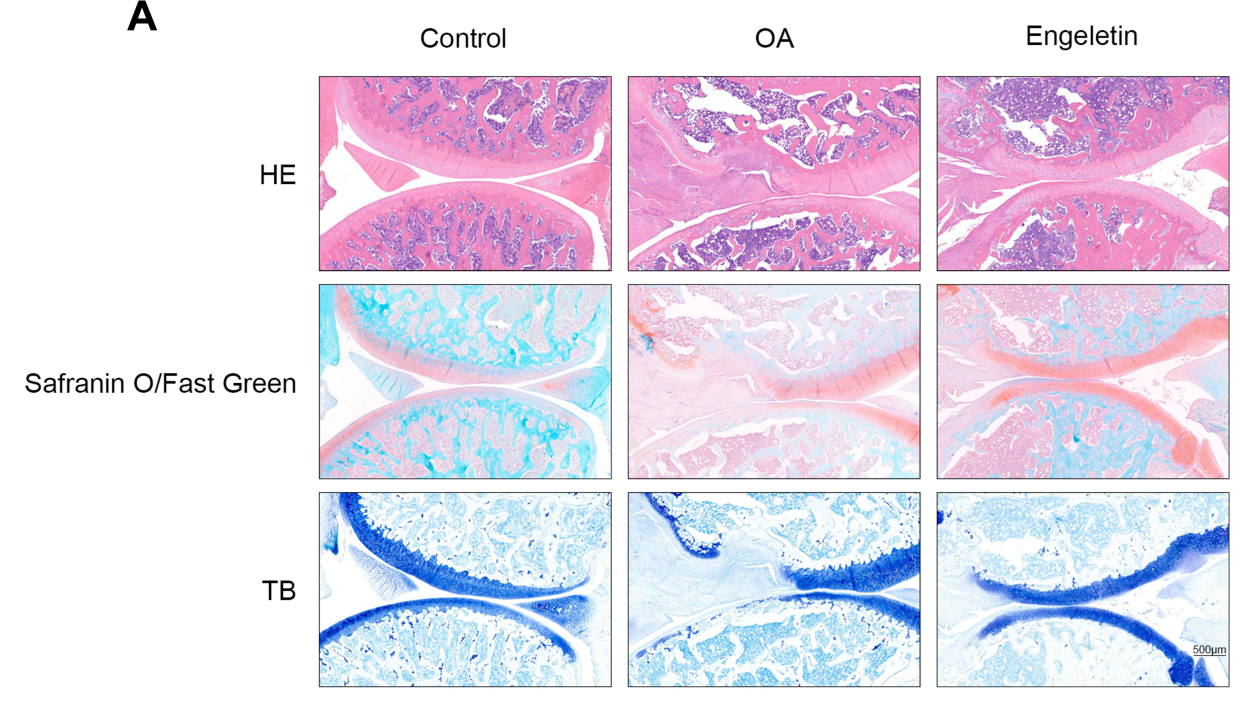

B

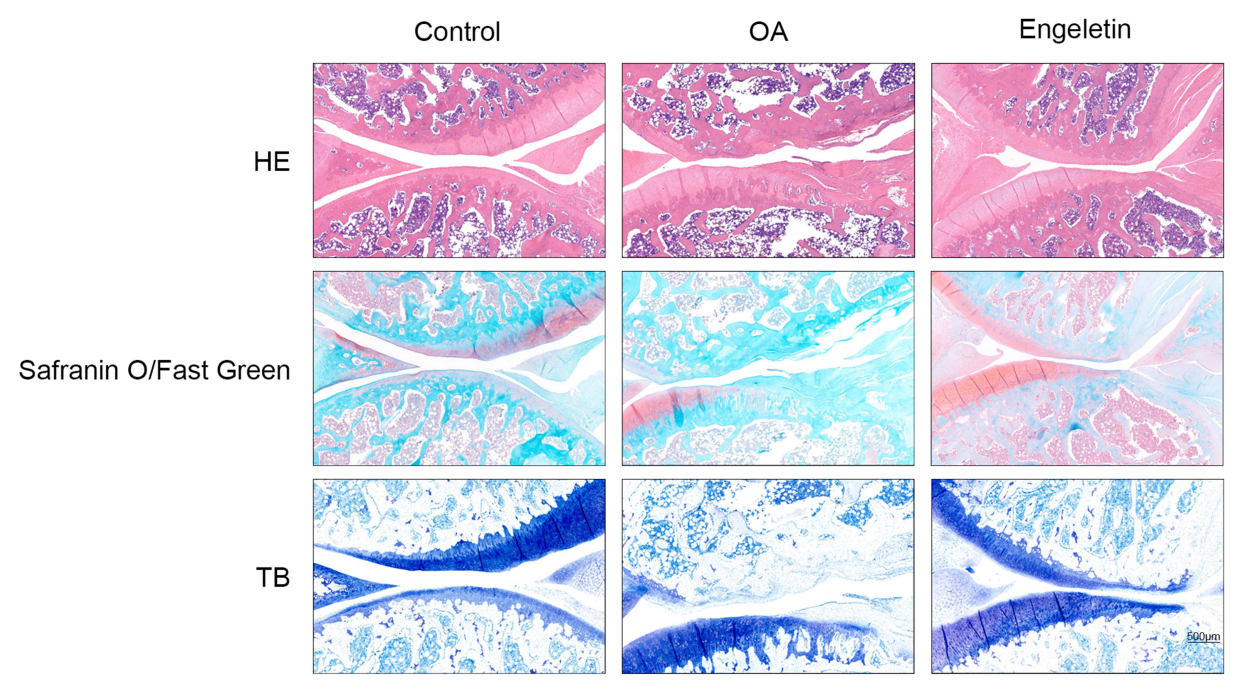

C

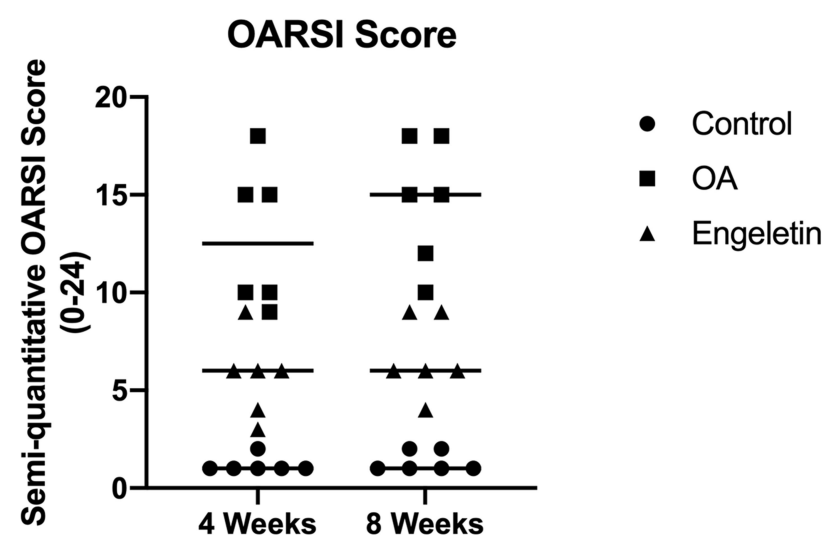

Figure 7 Histopathological analysis of the rat knee joint. (A, B) Representative hematoxylin-eosin, safranin O/fast green and toluidine blue staining images of rat knee cartilage from different groups after four (A) or eight (B) weeks of engeletin intervention. (C) Scatter plot of the OARSI scores of the knee cartilage from different groups. 


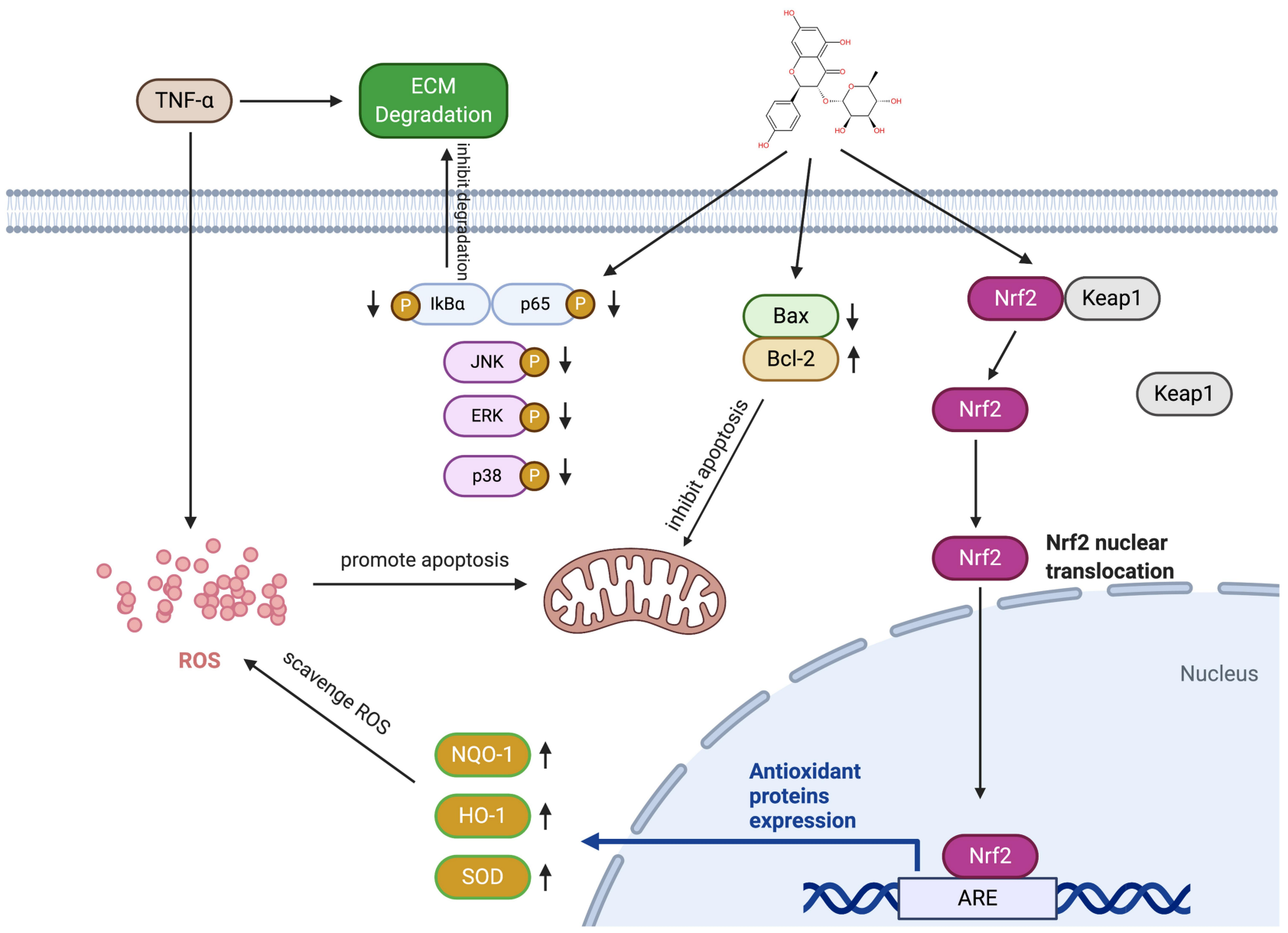

Figure 8 Schematic diagram of the mechanisms by which engeletin affects chondrocytes. TNF- $\alpha$ promotes apoptosis, ROS generation and ECM degradation in chondrocytes. Engeletin alleviates apoptosis by upregulating $\mathrm{Bcl}-2$ and downregulating Bax. Engeletin scavenges ROS by activating the Nrf2 pathway and inhibits ECM degradation by inhibiting the MAPK and NF- $\kappa B$ pathways.

far exceeding that of normal cartilage chondrocytes. ${ }^{30}$ This study focused on the association between engeletin and the intrinsic apoptotic pathway, which is modulated by mitochondria. Intrinsic stimuli, such as DNA damage and oxidative stress, upregulate the expression of proapoptotic proteins, including Bax and Bak, and downregulate the expression of antiapoptotic proteins, including Bcl-2 and Bcl-xL. An abnormal ratio of proapoptotic and antiapoptotic proteins results in mitochondrial pore formation and destroys mitochondrial membrane potential. Cytochrome $\mathrm{c}$ is then released from the mitochondria and activates the caspase cascade, initiating the apoptotic process. ${ }^{31-34}$ The present study showed that engeletin maintained mitochondrial membrane potential and alleviated chondrocyte apoptosis by upregulating $\mathrm{Bcl}-2$ expression and inhibiting $\mathrm{Bax}$ expression.

ROS are the initiators of the intrinsic apoptotic pathway. The mitochondrial permeability transition pore
(mPTP) plays an important role in modulating the redox homeostasis of cells. Low levels of ROS cause reversible opening of the mPTP, which helps eliminate ROS and maintain low levels. However, high levels of ROS cause irreversible opening of the MPTP. Irreversible opening may lead to ROS burst and result in the destruction of mitochondria, which initiates apoptosis. ${ }^{35}$ In addition, ROS inhibit the expression of ECM components and upregulate proinflammatory mediators. ${ }^{36}$ Various kinds of drugs have been discovered that are capable of scavenging intracellular ROS in chondrocytes. These drugs are divided into two categories: those that directly react with ROS and those that promote the expression of antioxidant proteins. ${ }^{37}$ Engeletin belongs to the latter category. This study proved that engeletin scavenged intracellular ROS by activating the Nrf2 pathway. The Nrf2 pathway is present in nearly all cell types and controls the antioxidant processes. Nrf2 binds with Kelch-like ECH-associated 
protein 1 (Keap1) in the cytoplasm if inactivated. Once activated by certain stimuli, Nrf2 detaches from Keap1, translocates to the nucleus and initiates the expression of several antioxidant proteins, including HO-1, NQO1, and SOD, which perform the scavenging processes. ${ }^{38-40}$

In addition to the Nrf2 pathway, the NF- $\mathrm{kB}$ and MAPK pathways are also involved in the progression of OA. I $\mathrm{B} \alpha$ and p65 are components of the NF- $\kappa B$ pathway and are activated through phosphorylation. In OA chondrocytes, activation of the NF- $\mathrm{KB}$ pathway is associated with upregulated expression of matrix catabolic enzymes, including MMP1, MMP2, MMP3, MMP7, MMP8, MMP9, MMP13, ADAMTS4 and ADAMTS5, and proinflammatory mediators, such as IL-1 $\beta$, IL- 6 , TNF- $\alpha$ and inducible nitric oxide synthase (iNOS). ${ }^{41-43}$ The MAPK pathway includes ERK, JNK and p38 and is also activated through phosphorylation. Activation of the MAPK pathway not only upregulates the expression of matrix catabolic enzymes and proinflammatory mediators but also contributes to the inhibition of other anabolic pathways, such as insulinlike growth factor 1 (IGF-1) and bone morphogenetic protein 7 (BMP7). ${ }^{38,44,45}$ The present study proved that engeletin inhibited the NF- $\mathrm{KB}$ and MAPK pathways, as shown by Western blotting. This inhibitory effect may be the underlying mechanism of the therapeutic effect of engeletin.

This study has two limitations. First, we only evaluated the effect of engeletin on each signaling pathway, but the interaction among pathways was not elucidated in this study. It remains unknown whether engeletin inhibits the MAPK and NF- $\kappa B$ pathways directly or by scavenging intracellular ROS and alleviating the inflammatory response. Second, we only examined changes in morphology and proteoglycan levels in cartilage by histopathological analysis. The in vivo study did not include elucidating the underlying mechanisms of engeletin, which will be evaluated through immunohistochemistry in the future.

\section{Conclusion}

To conclude, this study proved that engeletin ameliorated TNF- $\alpha$-induced ECM degradation, apoptosis and ROS generation in chondrocytes in vitro. The protective effect of engeletin occurred through the activation of the Nrf2 pathway and the inhibition of the NF- $\mathrm{KB}$ and MAPK pathways. In addition, engeletin alleviated ACLT-induced knee $\mathrm{OA}$ in a rat model in vivo. This study showed that engeletin may be a potential therapeutic drug for OA.

\section{Abbreviations}

OA, osteoarthritis; ROS, reactive oxygen species; IL-1 $\beta$, interleukin-1 $\beta$; IL-6, interleukin-6; PGE2, prostaglandin E2; TNF$\alpha$, tumor necrosis factor alpha; ECM, extracellular matrix; MMP, matrix metalloproteinase; ADAMTS, a disintegrin and metalloproteinase with thrombospondin motifs; Nrf2, nuclear factor erythroid 2-related factor 2; HO-1, heme oxygenase-1; SOD, superoxide dismutase; NQO1, NAD(P)H dehydrogenase, quinone 1 ; NF-kB, nuclear factor kappa-B; MAPK, mitogen-activated protein kinase; ACLT, anterior cruciate ligament transection; JNK, c-Jun N-terminal kinase; ERK, extracellular signal-regulated kinase; ІкB $\alpha$, inhibitor kappa $\mathrm{B}$ alpha; CCK8, Cell Counting Kit-8; SDS-PAGE, sodium dodecyl sulfatepolyacrylamide gel electrophoresis; PVDF, polyvinylidene difluoride; PI, propidium iodine; DCFH-DA, 2', $7^{\prime}$ dichlorofluorescein diacetate; HE, hematoxylin-eosin; TB, toluidine blue; mPTP, mitochondrial permeability transition pore; Keap1, Kelch-like ECH-associated protein 1.

\section{Acknowledgments}

We sincerely appreciate the support we received from central laboratory of Zhongshan Hospital.

\section{Funding}

This work was supported by the National Natural Science Foundation of China (No. 81702133, 81871742), Shanghai Hospital Development Center Emerging Advanced Technology Joint Research Project (No. SHDC12017107).

\section{Disclosure}

The authors report no conflicts of interest in this work.

\section{References}

1. James SL, Abate D, Abate KH. Global, regional, and national incidence, prevalence, and years lived with disability for 354 diseases and injuries for 195 countries and territories, 1990-2017: a systematic analysis for the Global Burden of Disease Study 2017. Lancet. 2018;392(10159):1789-1858.

2. Zhou JL, Deng S, Fang HS, Du XJ, Peng H, Hu QJ. Circular RNA circANKRD36 regulates Casz1 by targeting miR-599 to prevent osteoarthritis chondrocyte apoptosis and inflammation. J Cell Mol Med. 2020.

3. Li YS, Xiao WF, Luo W. Cellular aging towards osteoarthritis. Mech Ageing Dev. 2017;162:80-84.

4. Haseeb A, Haqqi TM. Immunopathogenesis of osteoarthritis. Clin immunol. 2013;146(3):185-196.

5. Hunter DJ, Bierma-Zeinstra S. Osteoarthritis. Lancet. 2019;393 (10182):1745-1759.

6. Jevsevar DS, Brown GA, Jones DL, et al. The American Academy of Orthopaedic Surgeons evidence-based guideline on: treatment of osteoarthritis of the knee, 2nd edition. J Bone Joint Surg Am. 2013;95(20):1885-1886. 
7. Lepetsos P, Papavassiliou AG. ROS/oxidative stress signaling in osteoarthritis. Biochim Biophys Acta. 2016;1862(4):576-591.

8. Khan NM, Haseeb A, Ansari MY, Devarapalli P, Haynie S, Haqqi TM. Wogonin, a plant derived small molecule, exerts potent anti-inflammatory and chondroprotective effects through the activation of ROS/ERK/Nrf2 signaling pathways in human Osteoarthritis chondrocytes. Free Radic Biol Med. 2017;106:288-301.

9. Chen Z, Zhong H, Wei J, et al. Inhibition of Nrf2/HO-1 signaling leads to increased activation of the NLRP3 inflammasome in osteoarthritis. Arthritis Res Ther. 2019;21(1):300.

10. Jiang C, Sun Z-M, Hu J-N, et al. Cyanidin ameliorates the progression of osteoarthritis via the Sirt6/NF- $\mathrm{kB}$ axis in vitro and in vivo. Food Funct. 2019;10(9):5873-5885. doi:10.1039/C9FO00742C

11. Arra M, Swarnkar G, Ke K, et al. LDHA-mediated ROS generation in chondrocytes is a potential therapeutic target for osteoarthritis. Nat Commun. 2020;11(1):3427. doi:10.1038/s41467-020-17242-0

12. Simon H-U, Haj-Yehia A, Levi-Schaffer F. Role of reactive oxygen species (ROS) in apoptosis induction. Apoptosis. 2000;5(5):415-418. doi:10.1023/A:1009616228304

13. Pacheco AGM, Branco A, Câmara CA. et al. Identification of flavonoids in Hymenaea martiana Hayne (Fabaceae) by HPLC-DAD-MSn analysis. Nat Prod Res;2019. 1-6. doi:10.1080/ 14786419.2019.1672062

14. Wang C, La L, Feng H, et al. Aldose reductase inhibitor engeletin suppresses pelvic inflammatory disease by blocking the phospholipase $\mathrm{C} /$ Protein Kinase $\mathrm{C}$-Dependent/NF- $\mathrm{KB}$ and MAPK Cascades. J Agric Food Chem. 2020;68(42):11747-11757. doi:10.1021/acs. jafc.0c05102

15. Wu H, Zhao G, Jiang K, Li C, Qiu C, Deng G. Engeletin Alleviates Lipopolysaccharide-Induced Endometritis in Mice by Inhibiting TLR4-mediated NF-kB Activation. J Agric Food Chem. 2016;64 (31):6171-6178. doi:10.1021/acs.jafc.6b02304

16. Ye W, Chen R, Sun W, et al. Determination and pharmacokinetics of engeletin in rat plasma by ultra-high performance liquid chromatography with tandem mass spectrometry. J Chromatogr B Analyt Technol Biomed Life Sci. 2017;1060:144-149. doi:10.1016/j. jchromb.2017.06.018

17. Huang Z, Ji H, Shi J, Zhu X, Zhi Z. Engeletin Attenuates Aß1-42Induced Oxidative Stress and Neuroinflammation by Keap1/Nrf2 Pathway. Inflammation. 2020;43(5):1759-1771. doi:10.1007/s10753020-01250-9

18. Liu T, Li Y, Sun J, Tian G, Shi Z. Engeletin suppresses lung cancer progression by inducing apoptotic cell death through modulating the XIAP signaling pathway: a molecular mechanism involving ER stress. Biomed Pharmacother. 2000;5:110221. doi:10.1016/j. biopha.2020.110221

19. Jiang $X$, Chen L, Zhang Z, Sun Y, Wang X, Protective WJ. Therapeutic Effects of Engeletin on LPS-Induced Acute Lung Injury. Inflammation. 2018;41(4):1259-1265.

20. Khan NM, Ahmad I, Haqqi TM. Nrf2/ARE pathway attenuates oxidative and apoptotic response in human osteoarthritis chondrocytes by activating ERK1/2/ELK1-P70S6K-P90RSK signaling axis. Free Radic Biol Med. 2018;116:159-171.

21. Yang G, Sun S, Wang J, et al. S-Allylmercaptocysteine Targets Nrf2 in Osteoarthritis Treatment Through NOX4/NF-кB Pathway. Drug Des Devel Ther. 2020;14:4533-4546.

22. Xue XH, Xue JX, Hu W, Shi FL, Yang Y. Nomilin targets the Keap1-Nrf2 signalling and ameliorates the development of osteoarthritis. J Cell Mol Med. 2020;24(15):8579-8588.

23. Xu K, Ma C, Xu L, et al. Polygalacic acid inhibits MMPs expression and osteoarthritis via Wnt/ $\beta$-catenin and MAPK signal pathways suppression. Int Immunopharmacol. 2018;63:246-252.

24. Hu ZC, Xie ZJ, Tang Q, et al. Hydroxysafflor yellow A (HSYA) targets the NF- $\mathrm{KB}$ and MAPK pathways and ameliorates the development of osteoarthritis. Food Funct. 2018;9(8):4443-4456.
25. Tian Q, Wang G, Zhang Y, et al. Engeletin inhibits Lipopolysaccharide/d-galactosamine-induced liver injury in mice through activating PPAR- $\gamma$. J Pharmacol Sci. 2019;140(3):218-222.

26. Feng H, He Y, La L, et al. The flavonoid-enriched extract from the root of Smilax china L. inhibits inflammatory responses via the TLR-4-mediated signaling pathway. $J$ Ethnopharmacol. 2020;256:112785.

27. Livak KJ, Schmittgen TD. Analysis of relative gene expression data using real-time quantitative PCR and the 2(-Delta Delta C(T)) Method. Methods. 2001;25(4):402-408.

28. Pritzker KP, Gay S, Jimenez SA, et al. Osteoarthritis cartilage histopathology: grading and staging. Osteoarthritis Cartilage. 2006;14(1):13-29.

29. Tschaikowsky M, Selig M, Brander S, Balzer BN, Hugel T, Rolauffs B. Proof-of-concept for the detection of early osteoarthritis pathology by clinically applicable endomicroscopy and quantitative AI-supported optical biopsy. Osteoarthritis Cartilage. 2020.

30. Sharif M, Whitehouse A, Sharman P, Perry M, Adams M. Increased apoptosis in human osteoarthritic cartilage corresponds to reduced cell density and expression of caspase-3. Arthritis Rheum. 2004;50 (2):507-515.

31. Lin VS, Xu ZF, Huang DCS, Thijssen R. BH3 Mimetics for the Treatment of B-Cell Malignancies-Insights and Lessons from the Clinic. Cancers. 2020;12:11.

32. Lee JH, Park SY, Hwang W, et al. Isoharringtonine induces apoptosis of non-small cell lung cancer cells in tumorspheroids via the intrinsic pathway. Biomolecules. 2020;10:11.

33. Kapoor I, Bodo J, Hill BT, Hsi ED, Targeting AA. BCL-2 in B-cell malignancies and overcoming therapeutic resistance. Cell Death Dis. 2020;11(11):941.

34. Yapasert R, Sripanidkulchai B, Teerachaisakul M, Banchuen K, Banjerdpongchai R. Anticancer effects of a traditional Thai herbal recipe Benja Amarit extracts against human hepatocellular carcinoma and colon cancer cell by targeting apoptosis pathways. J Ethnopharmacol. 2020;254:112732.

35. Zorov DB, Juhaszova M, Sollott SJ. Mitochondrial reactive oxygen species (ROS) and ROS-induced ROS release. Physiol Rev. 2014;94 (3):909-950.

36. Goldring MB, Berenbaum F. The regulation of chondrocyte function by proinflammatory mediators: prostaglandins and nitric oxide. Clin Orthop Relat Res. 2004;427 Suppl:S37-S46.

37. Kavanaugh TE, Dailing EA, Cho H, Hasty KA, Duvall CL. Development of optimized copolymers and delivery formulations to scavenge reactive oxygen species and prevent joint damage from post-traumatic osteoarthritis. Osteoarthritis Cartilage. 2017;25:S265-S266.

38. Bolduc JA, Collins JA, Loeser RF. Reactive oxygen species, aging and articular cartilage homeostasis. Free Radic Biol Med. 2019;132:73-82.

39. Cai D, Yin S, Yang J, Jiang Q, Cao W. Histone deacetylase inhibition activates Nrf2 and protects against osteoarthritis. Arthritis Res Ther. 2015;17:269.

40. Yang J, Song X, Feng Y, et al. Natural ingredients-derived antioxidants attenuate $\mathrm{H}(2) \mathrm{O}(2)$-induced oxidative stress and have chondroprotective effects on human osteoarthritic chondrocytes via Keap1/Nrf2 pathway. Free Radic Biol Med. 2020;152:854-864.

41. Rigoglou S, Papavassiliou AG. The NF- $\mathrm{kB}$ signalling pathway in osteoarthritis. Int J Biochem Cell Biol. 2013;45(11):2580-2584.

42. Saito T, Tanaka S. Molecular mechanisms underlying osteoarthritis development: notch and NF-кB. Arthritis Res Ther. 2017;19(1):94.

43. $\mathrm{Lu} \mathrm{H}, \mathrm{Fu} \mathrm{C}$, Kong $\mathrm{S}$, et al. Maltol prevents the progression of osteoarthritis by targeting PI3K/Akt/NF- $\mathrm{kB}$ pathway: in vitro and in vivo studies. J Cell Mol Med. 2020;25(1): 499-509.

44. Nazli SA, Loeser RF, Chubinskaya S, Willey JS, Yammani RR. High fat-diet and saturated fatty acid palmitate inhibits IGF-1 function in chondrocytes. Osteoarthritis Cartilage. 2017;25(9):1516-1521.

45. Sun Y, Zuo Z, Kuang Y. An emerging target in the battle against osteoarthritis: macrophage polarization. Int J Mol Sci. 2020;21 (22):8513. 


\section{Publish your work in this journal}

The Journal of Inflammation Research is an international, peerreviewed open-access journal that welcomes laboratory and clinical findings on the molecular basis, cell biology and pharmacology of inflammation including original research, reviews, symposium reports, hypothesis formation and commentaries on: acute/chronic inflammation; mediators of inflammation; cellular processes; molecular mechanisms; pharmacology and novel anti-inflammatory drugs; clinical conditions involving inflammation. The manuscript management system is completely online and includes a very quick and fair peerreview system. Visit http://www.dovepress.com/testimonials.php to read real quotes from published authors. 